\title{
Stokes waves in water with a non-flat bed
}

\author{
A. Constantin $\dagger$ \\ Department of Mathematics, King's College London, Strand, London WC2R 2LS, UK \\ Faculty of Mathematics, University of Vienna, Oskar-Morgenstern-Platz 1, 1090 Vienna, Austria
}

(Received 31 July 2013; revised 8 October 2013; accepted 27 November 2013; first published online 8 January 2014)

We investigate the relevance of Stokes waves for the propagation of ocean swell in the absence of strong currents. By providing estimates for the depth of the near-surface layer to which the main effects of a Stokes flow are confined, we show that windgenerated uniform wave trains can be modelled as Stokes waves over a fictitious flat bed, immersed in the water. Throughout the lower parts of this layer the deviations of the flow from a pure current are negligible.

Key words: surface gravity waves, waves/free-surface flows

\section{Introduction}

Meteorological disturbances operating over considerable distances often result in periodic travelling surface ocean waves that persist after the disturbance has abated, almost without losing the acquired energy. These wave patterns, termed swell, are representative of the simplest type of non-trivial surface wave: a periodic profile that propagates at constant speed in a fixed direction. Swell from large storms occurring at moderate and high latitudes in the Atlantic Ocean and in the Pacific Ocean often travels thousands of $\mathrm{km}$ over the ocean. It is customary to model swell by means of a linearization approach but linear deep-water wave theory only accommodates sinusoidal travelling waves (see §3). Consequently, the sharp crests and flat troughs that are characteristic for swell, see e.g. the photographs of swell in Constantin (2011), are not captured by linear theory. On the other hand, nonlinear Stokes waves - the exact meaning of the term being defined at the beginning of $\S 4-$ present this type of feature and the available analytical, numerical, and experimental studies of Stokes waves are by now quite detailed, see the discussions in Buffoni \& Toland (2003), Clamond (2012) and Umeyama (2012). However, Stokes wave theory is suitable for waves propagating in water that is either infinitely deep or has a flat bed, see the discussions in Toland (1996), Henry (2008) and Constantin (2013).

There are extensive ocean regions with a flat bed: abyssal plains are the flattest areas on Earth, with depth variations commonly in the range of $10-100 \mathrm{~cm}$ per km of horizontal distance. They are found in all major sea and ocean basins, being vast sediment-covered regions of the sea floor formed by the blanketing of a pre- 
existent irregular sea bed by accumulated land-derived sediment. For example, the Sohm Plain in the North Atlantic has an area of $\sim 900000 \mathrm{~km}^{2}$. However, deep-sea regions presenting high variations of the bed topography are also quite common. The relevance of Stokes waves in such a setting is suggested by field data indicating that in the absence of strong currents, the main effects of the flow beneath the swell are confined to a near-surface layer whose depth rarely exceeds $250 \mathrm{~m}$. Thus swell in deep water without strong underlying currents might be modelled as a Stokes wave propagating over a fictitious flat bed, beneath which there is practically no motion. Our aim is to substantiate this observation. We provide some estimates showing that the flow beneath a Stokes wave has a rapid decay. Our theoretical conclusions are applicable to the typical setting of swell in the Atlantic Ocean and in the Pacific Ocean.

\section{Preliminaries}

In our deep-water setting we allow for strong variations of the bed topography since our contention is that the flow is practically confined to a near-surface layer beneath which the water can be regarded as being still. In the near-surface layer the flow is induced by a two-dimensional water wave, so that it suffices to investigate its characteristics in a cross-section oriented towards the direction of wave propagation. We choose Cartesian coordinates $(X, Y)$ with the $X$-axis pointing in the direction of wave propagation and the $Y$-axis pointing vertically upwards. Let $Y=0$ be the mean water level. We denote by $(U(X-c T, Y), V(X-c T, Y))$ the velocity field of the two-dimensional flow propagating in the $X$-direction and let $Y=\eta(X-c T)$ be the free surface and $Y=-d$ be the lower boundary of the near-surface layer in which the main effects of the flow are noticeable. Here $c>0$ is the (constant) speed of wave propagation. A formula for the speed in terms of the wave parameters can be easily obtained within the setting of linear theory cf. (3.11) in $\S 3$; for its validity without performing approximations, that is, within the nonlinear setting, we refer to the discussion in Constantin (2011). Under the physically realistic assumptions of inviscid flow of water with constant density, the equation of mass conservation and the condition of irrotational flow are

$$
U_{X}+V_{Y}=0
$$

and

$$
U_{Y}=V_{X},
$$

respectively, while the equation of motion is Euler's equation

$$
\left.\begin{array}{l}
(U-c) U_{X}+V U_{Y}=-\frac{P_{X}}{\rho}, \\
(U-c) V_{X}+V V_{Y}=-\frac{P_{Y}}{\rho}-g,
\end{array}\right\}
$$

where $P(X-c T, Y)$ is the pressure, $\rho$ is the density and $g$ is the (constant) acceleration due to gravity. The boundary conditions associated with the above equations are the kinematic boundary conditions

$$
\begin{gathered}
V=(U-c) \eta_{X} \quad \text { on } Y=\eta(X-c T), \\
V=0 \quad \text { on } Y=-d,
\end{gathered}
$$


and the dynamic boundary condition

$$
P=P_{a t m} \quad \text { on } Y=\eta(X-c T),
$$

where $P_{a t m}$ is the (constant) atmospheric pressure at the surface. Beneath the nearsurface layer lying above $Y=-d$, our contention is that the flow effects are negligible, so that the water is practically still. For the physical relevance of the governing equations (2.1)-(2.6) in the modelling of swell, we refer to the discussion in Constantin (2011).

\section{Inadequacy of linear theory}

The classical approach to gain insight into the dynamics of the governing equations (2.1)-(2.6) is by means of linearization. If $a$ is the maximal amplitude of the wave and $\lambda$ is the wavelength, introducing the perturbation $p$ of the pressure $P$ relative to the hydrostatic pressure by means of

$$
P(X-c T, Y)=P_{a t m}-\rho g Y+\rho g a p(X-c T, Y),
$$

and performing (see e.g. Clamond 1999 or Constantin \& Johnson 2008) the standard non-dimensionalization and scaling

$$
X=\lambda x, \quad Y=d y, \quad T=\frac{\lambda}{\sqrt{g d}} t, \quad \eta=a h, \quad U=\frac{a \sqrt{g d}}{d} u, \quad V=\frac{a \sqrt{g d}}{\lambda} v,
$$

the governing equations (2.1)-(2.6) are transformed into the system

$$
\begin{aligned}
& u_{x}+v_{y}=0 \text { for }-1<y<\varepsilon h, \\
& u_{y}=\delta^{2} v_{x} \text { for }-1<y<\varepsilon h, \\
& \left(\varepsilon u-c_{0}\right) u_{x}+\varepsilon v u_{y}=-p_{x} \quad \text { for }-1<y<\varepsilon h, \\
& \delta^{2}\left\{\left(\varepsilon u-c_{0}\right) v_{x}+\varepsilon v v_{y}\right\}=-p_{y} \text { for }-1<y<\varepsilon h, \\
& v=\left(\varepsilon u-c_{0}\right) h_{x} \quad \text { on } y=\varepsilon h, \\
& v=0 \text { on } y=-1, \\
& p=h \quad \text { on } y=\varepsilon h,
\end{aligned}
$$

where $c_{0}=c / \sqrt{g d}$ is the non-dimensional wave speed, and

$$
\varepsilon=\frac{a}{d}, \quad \delta=\frac{d}{\lambda}
$$

are the amplitude and shallowness parameters, respectively. The shallow-water regime $\delta \ll 1$ is too restrictive since we would like to allow wavelengths $\lambda$ of the same order as the average depth $d$ of the near-surface layer. Consequently, we keep $\delta$ fixed and consider the linearized problem

$$
\begin{array}{r}
u_{x}+v_{y}=0 \quad \text { for }-1<y<0, \\
u_{y}=\delta^{2} v_{x} \quad \text { for }-1<y<0, \\
c_{0} u_{x}=p_{x} \quad \text { for }-1<y<0, \\
c_{0} \delta^{2} v_{x}=p_{y} \quad \text { for }-1<y<0, \\
v=-c_{0} h_{x} \quad \text { on } y=0, \\
v=0 \quad \text { on } y=-1, \\
p=h \quad \text { on } y=0,
\end{array}
$$


obtained from (3.3) by letting $\varepsilon \rightarrow 0$. The system (3.5) describes waves of small amplitude. We seek symmetric waves: $h$ is even in the $x$-variable, with $u$ and $p$ also even while $v$ is odd. The first two equations yield $\delta^{2} v_{x x}+v_{y y}=0$ for $-1<y<0$. Taking into account the boundary condition on $y=-1$ in (3.5), one can see that the Fourier series expansion of the function $v$ has to be of the form

$$
v(x, y)=\sum_{n \geqslant 1} v_{n} \sinh [2 \pi n \delta(y+1)] \sin (2 \pi n x), \quad-1 \leqslant y \leqslant 0,
$$

for some real coefficients $v_{n}$. The first two equations in (3.5) yield

$$
u(x, y)=\gamma+\delta \sum_{n \geqslant 1} v_{n} \cosh [2 \pi n \delta(y+1)] \cos (2 \pi n x), \quad-1 \leqslant y \leqslant 0,
$$

for some constant $\gamma$, representing the strength of the uniform wave-induced current (see $\S 4$ ). The absence of a current corresponds to $\gamma=0$, which is the appropriate choice for our setting. Now the third and fourth equations in (3.5) show that $p=p_{0}+c_{0} u$ throughout the strip $-1 \leqslant y \leqslant 0$, for some constant $p_{0}$. Since zero mean water level translates into the average of $h$ over one period being zero, the last relation in (3.5) forces $p_{0}=0$ and yields

$$
h(x)=c_{0} \delta \sum_{n \geqslant 1} v_{n} \cosh [2 \pi n \delta] \cos (2 \pi n x) .
$$

The fifth equation in (3.5) can therefore be expressed as

$$
\sum_{n \geqslant 1} v_{n} \sinh [2 \pi n \delta] \sin (2 \pi n x)=2 \pi c_{0}^{2} \delta \sum_{n \geqslant 1} n v_{n} \cosh [2 \pi n \delta] \sin (2 \pi n x) .
$$

This identity holds only if $c_{0}^{2}=\tanh (2 \pi n \delta) / 2 \pi n \delta$ whenever $v_{n} \neq 0$. The function $s \mapsto \tanh (s) / s$ being strictly decreasing for $s>0$, there is at most one integer $n \geqslant 1$ for which $v_{n} \neq 0$. This proves that superpositions of Fourier modes are not possible: with principal unit period, the surface wave is of the form $h(x)=A \cos (2 \pi x)$, obtained by choosing $v_{n}=0$ for $n \geqslant 2$ and $A=c_{0} \delta v_{1} \cosh (2 \pi \delta)$. The corresponding full solution, written in physical variables, is

$$
\left.\begin{array}{l}
\eta(X-c T)=\varepsilon d A \cos \left(\frac{2 \pi(X-c T)}{\lambda}\right), \\
U(X-c T, Y)=\frac{\varepsilon g d A}{c} \frac{\cosh \left(\frac{2 \pi(Y+d)}{\lambda}\right)}{\cosh \left(\frac{2 \pi d}{\lambda}\right)} \cos \left(\frac{2 \pi(X-c T)}{\lambda}\right), \\
V(X-c T, Y)=\frac{\varepsilon g d A}{c} \frac{\sinh \left(\frac{2 \pi(Y+d)}{\lambda}\right)}{\cosh \left(\frac{2 \pi d}{\lambda}\right)} \sin \left(\frac{2 \pi(X-c T)}{\lambda}\right), \\
P(X-c T, Y)=P_{a t m}-\rho g Y+\varepsilon \rho g d A \frac{\cosh \left(\frac{2 \pi(Y+d)}{\lambda}\right)}{\cosh \left(\frac{2 \pi d}{\lambda}\right)} \cos \left(\frac{2 \pi(X-c T)}{\lambda}\right),
\end{array}\right\}
$$


where

$$
c=\sqrt{\frac{g \lambda}{2 \pi} \tanh \left(\frac{2 \pi d}{\lambda}\right)} .
$$

In (3.10), note the decay rate of the vertical component of the velocity field as one descends in the fluid beneath the free surface towards the lower boundary of the near-surface layer.

Due to the fact that only sinusoidal wave profiles are permissible, linear theory fails to capture the typical swell pattern: almost flat near the troughs and with pronounced elevations near the crest, see e.g. the photographs in Constantin (2011). Consequently, linear theory is grossly inaccurate in some respects and nonlinear effects should be accounted for.

\section{The flow beneath a Stokes wave}

We call a Stokes wave a smooth travelling wave solution to the governing equations (2.1)-(2.6), for which $\eta, U, V$ are all periodic in the $x$-variable, with the functions $\eta$, $U$ even and $V$ odd in the $X$-variable. Moreover, the wave profile $\eta$ should be strictly monotone between consecutive crests and troughs, and symmetric. The existence theory of Stokes waves and the study of the wave profile are by now quite advanced. For example, it is known that $\left|\eta_{X}\right| \leqslant 1$, cf. Amick (1987), and the Fourier series expansion of the free surface presents interesting features, cf. Plotnikov \& Toland (2002). While often the term 'Stokes wave' is used for approximations obtained by means of a perturbative scheme, we refer to an exact solution. We recall that under the assumption of small wave steepness, $k a \ll 1$, Stokes (1847) derived a second-order expansion for progressive periodic waves of permanent form. For engineering purposes, a widely used fifth-order expansion is due to Fenton (1985). However, the Stokes expansion and its extensions are generally not reliable for waves of moderate and large amplitude, cf. the discussion in Drennan, Hui \& Tenti (1992), while in the shallowwater regime $d \ll \lambda$ its predictions are not accurate even for waves of small amplitude. To prevent such shortcomings, we consider exact solutions without approximations. In particular, our conclusions are valid for all wavelengths, including periodic travelling waves in shallow water.

An important aspect of the flow beneath a Stokes wave concerns the underlying current. We assume that in a frame of reference moving at the constant wave speed $c$, the wave crest is located on the line $X=0$, called the crest line, so that the wave troughs lie on the lines $X= \pm \lambda / 2$, called trough lines. Applying the divergence theorem to the vector field $(V,-U)$ in the rectangular domain delimited laterally by the trough lines $X= \pm \lambda / 2$, bounded above by the trough level $Y=Y_{0}$ with $Y_{0}=\eta(\lambda / 2)<0$, and below by the lower boundary $Y=-d$ of the near-surface layer, the identity

$$
\int_{-\lambda / 2}^{\lambda / 2} U(X, Y) \mathrm{d} X=\int_{-\lambda / 2}^{\lambda / 2} U(X,-d) \mathrm{d} X, \quad-d \leqslant Y \leqslant Y_{0},
$$

emerges. The physical interpretation of this identity is that

$$
\kappa=\frac{1}{\lambda} \int_{-\lambda / 2}^{\lambda / 2} U(X,-d) \mathrm{d} X
$$


represents the uniform underlying current, see the discussions in Constantin \& Strauss (2010) or in Constantin (2013). In our setting, characterized by the absence of a current, we have $\kappa=0$. In these circumstances, it is known, cf. Constantin (2006) that

$$
V(X, Y)>0 \text { for } 0<X<\lambda / 2,-d<Y \leqslant \eta(X),
$$

with $V(0, Y)=0$ for $Y \in[-d, \eta(0)]$ and $V(\lambda / 2, Y)=0$ for $Y \in[-d, \eta(\lambda / 2)]$, while

$$
U(X, Y)<c \text { throughout the fluid domain }-d \leqslant Y \leqslant \eta(X),
$$

with $U$ attaining its maximum value at the wave crest and its minimum value at the wave trough. Moreover, $U$ is strictly decreasing/increasing as we descend into the fluid along the crest/trough line, with the function $X \mapsto U(X,-d)$ strictly decreasing on $[0, \lambda / 2]$. Since $\kappa=0$, there exists a unique point $X_{0} \in(0, \lambda / 2)$ with $U\left(X_{0},-d\right)=0$.

We will now prove some estimates that quantify the decay of the flow as one descends in the fluid beneath the free surface. In this context, it is useful to note that (2.4) and (2.5) provide us with the flow-invariant integral

$$
p_{0}=\int_{-d}^{\eta(X)}(U(X, Y)-c) \mathrm{d} Y, \quad-\lambda / 2 \leqslant X \leqslant \lambda / 2,
$$

representing the relative mass flux. Note that $p_{0}<0$, due to (4.4). The monotonicity properties of $U$ mentioned above yield

$$
c[\eta(0)+d]>[c-U(0,-d)][\eta(0)+d]>\int_{-d}^{\eta(0)}(c-U(0, Y)) \mathrm{d} Y=\left|p_{0}\right|,
$$

and

$$
\begin{aligned}
c[\eta(\lambda / 2)+d] & <[c-U(\lambda / 2,-d)][\eta(\lambda / 2)+d] \\
& <\int_{-d}^{\eta(\lambda / 2)}(c-U(\lambda / 2, Y)) \mathrm{d} Y=\left|p_{0}\right| .
\end{aligned}
$$

Consequently

$$
\frac{\left|p_{0}\right|}{\eta(0)+d}<c-U(0,-d)<c<c-U(\lambda / 2,-d)<\frac{\left|p_{0}\right|}{\eta(\lambda / 2)+d},
$$

so that

$$
\begin{aligned}
0 & <[c-U(\lambda / 2,-d)]-[c-U(0,-d)]<\frac{\left|p_{0}\right|}{\eta(\lambda / 2)+d}-\frac{\left|p_{0}\right|}{\eta(0)+d} \\
& =\frac{\left|p_{0}\right|[\eta(0)-\eta(\lambda / 2)]}{[\eta(\lambda / 2)+d][\eta(0)+d]} \leqslant \frac{c[\eta(0)-\eta(\lambda / 2)]}{\eta(\lambda / 2)+d},
\end{aligned}
$$

using (4.6) in the last step. The above monotonicity and periodicity properties of $U$ ensure that the variation of $U$ along the lower boundary $Y=-d$ of the near-surface layer is precisely $[U(0,-d)-U(\lambda / 2,-d)]>0$. We deduce that

$$
U(0,-d)-U(\lambda / 2,-d)=\sup _{X \in[-\lambda / 2, \lambda / 2]}\{|U(X,-d)-U(X, 0)|\}<\frac{c[\eta(0)-\eta(\lambda / 2)]}{\eta(\lambda / 2)+d} .
$$

We now investigate the decay of the flow field throughout the near-surface layer as the depth increases. Note that $V$ being harmonic and odd in the $X$-variable in the domain $\Omega=\{(X, Y):-\lambda / 2 \leqslant X \leqslant \lambda / 2,-d \leqslant Y \leqslant \eta(X)\}$, with $V(X,-d)=0$ for 
all $X \in[-\lambda / 2, \lambda / 2]$ in view of (2.5), it will admit a Fourier series representation of the form

$$
V(X, Y)=\sum_{n \geqslant 1} \alpha_{n} \sinh [k n(Y+d)] \sin (k n X), \quad(X, Y) \in \Omega,
$$

where $k=2 \pi / \lambda$ is the wavenumber. Moreover, the validity of (4.3) along $Y=\eta(X)$, together with the fact that $V(0, \eta(0))=V(\lambda / 2, \eta(\lambda / 2))=0$ while for $X \in\{0, \lambda / 2\}$ we have that $\partial_{X}[V(X, \eta(X))]=V_{X}(X, \eta(X))=U_{Y}(X, \eta(X)) \neq 0$ due to (2.2) and the strict monotonicity of $U$ along the crest and trough lines, ensure the existence of some $m_{+}>m_{-}>0$ with

$$
0<m_{-} \sinh [k(\eta(0)+d)] \sin (k X)<V(X, \eta(X))<m_{+} \sinh \left[k\left(Y_{0}+d\right)\right] \sin (k X)
$$

for $X \in(0, \lambda / 2)$. Consider now the harmonic functions

$$
w_{ \pm}(X, Y)=V(X, Y)-m_{\mp} \sinh [k(Y+d)] \sin (k X)
$$

in the domain $\Omega_{+}=\{(X, Y): 0 \leqslant X \leqslant \lambda / 2,-d \leqslant Y \leqslant \eta(X)\}$. The choice of the constants $m_{ \pm}$ensures that $w_{-}(X, Y) \leqslant 0 \leqslant w_{+}(X, Y)$ on the entire boundary of $\Omega_{+}$. The maximum principle for harmonic functions yields the validity of these inequalities throughout $\Omega_{+}$. Consequently

$$
0 \leqslant m_{-} \sinh [k(Y+d)] \sin (k X) \leqslant V(X, Y) \leqslant m_{+} \sinh [k(Y+d)] \sin (k X)
$$

throughout $\Omega_{+}$. The estimate of the decay rate for the vertical component of the velocity field provided by (4.14) is precisely that predicted by linear theory, cf. (3.10).

Regarding the horizontal component of the velocity field, due to (2.1), (2.2) and the fact that $\kappa=0$, it admits the Fourier series representation

$$
U(X, Y)=\sum_{n \geqslant 1} \alpha_{n} \cosh [k n(Y+d)] \cos (k n X), \quad(X, Y) \in \Omega .
$$

Let $\|f\|_{L^{2}[0, \lambda / 2]}^{2}=\int_{0}^{\lambda / 2} f^{2}(X) \mathrm{d} X$ for a square-integrable function $f$. From (4.15) we obtain that

$$
\|U(\cdot, Y)\|_{L^{2}[0, \lambda / 2]}^{2}=\frac{\lambda}{4} \sum_{n \geqslant 1} \alpha_{n}^{2} \cosh ^{2}[k n(Y+d)], \quad-d \leqslant Y \leqslant Y_{0} .
$$

Consequently

$$
\frac{\|U(\cdot, Y)\|_{L^{2}[0, \lambda / 2]}}{\|U(\cdot,-d)\|_{L^{2}[0, \lambda / 2]}} \geqslant \cosh [k(Y+d)], \quad-d \leqslant Y \leqslant Y_{0} .
$$

This estimates the growth of $U$ above the flat bottom of the near-surface layer. On the other hand, for $Y \in\left[-d, Y_{0}\right]$ we have

$$
\begin{aligned}
\|U(\cdot, Y)\|_{L^{2}[0, \lambda / 2]}^{2} & =\frac{\lambda}{4} \sum_{n \geqslant 1} \alpha_{n}^{2} \cosh ^{2}[k n(Y+d)]=\frac{\lambda}{4} \sum_{n \geqslant 1} \alpha_{n}^{2}+\frac{\lambda}{4} \sum_{n \geqslant 1} \alpha_{n}^{2} \sinh ^{2}[k n(Y+d)] \\
& =\|U(\cdot,-d)\|_{L^{2}[0, \lambda / 2]}^{2}+\|V(\cdot, Y)\|_{L^{2}[0, \lambda / 2]}^{2}
\end{aligned}
$$

so that (4.14) yields

$$
0 \leqslant\|U(\cdot, Y)\|_{L^{2}[0, \lambda / 2]}^{2}-\|U(\cdot,-d)\|_{L^{2}[0, \lambda / 2]}^{2} \leqslant \frac{\lambda m_{+}^{2}}{4} \sinh ^{2}[k(Y+d)], \quad-d \leqslant Y \leqslant Y_{0} .
$$


It is also possible to derive an estimate throughout the near-surface layer for the variation of $U$ evaluated beneath the crest and beneath the trough. For this, note that

$$
U(0, Y)-U(\lambda / 2, Y)=2 \sum_{n=2 j+1: j \geqslant 0} \alpha_{n} \cosh [k n(Y+d)], \quad Y \in\left[-d, Y_{0}\right]
$$

while

$$
\int_{0}^{\lambda / 2} \frac{V(X, Y)}{\sin (k X)} \mathrm{d} X=\frac{\lambda}{2} \sum_{n=2 j+1: j \geqslant 0} \alpha_{n} \sinh [k n(Y+d)], \quad Y \in\left[-d, Y_{0}\right] .
$$

The first relation follows directly from (4.15), while the second is a consequence of (4.11) in combination with the identity

$$
\int_{0}^{\lambda / 2} \frac{\sin (k n X)}{\sin (k X)} \mathrm{d} X= \begin{cases}0 & \text { if } n \geqslant 1 \text { is even } \\ \lambda / 2 & \text { if } n \geqslant 1 \text { is odd. }\end{cases}
$$

The validity of (4.22) is easily checked by an inductive argument: for $n=1$ and $n=2$ the formula is immediate, while for $n \geqslant 3$ we have

$$
\begin{aligned}
\int_{0}^{\lambda / 2} \frac{\sin (k n X)}{\sin (k X)} \mathrm{d} X= & \int_{0}^{\lambda / 2} \frac{\sin [k(n-1) X] \cos (k X)}{\sin (k X)} \mathrm{d} X+\int_{0}^{\lambda / 2} \cos [k(n-1) X] \mathrm{d} x \\
= & \int_{0}^{\lambda / 2} \frac{\sin [k(n-1) X] \cos (k X)}{\sin (k X)} \mathrm{d} X \\
= & \int_{0}^{\lambda / 2} \frac{\sin [k(n-2) X] \cos ^{2}(k X)}{\sin (k X)} \mathrm{d} X \\
& +\int_{0}^{\lambda / 2} \cos [k(n-2) X] \cos (k X) \mathrm{d} X \\
= & \int_{0}^{\lambda / 2} \frac{\sin [k(n-2) X]}{\sin (k X)} \mathrm{d} X+\int_{0}^{\lambda / 2} \cos [k(n-1) X] \mathrm{d} X \\
= & \int_{0}^{\lambda / 2} \frac{\sin [k(n-2) X]}{\sin (k X)} \mathrm{d} X .
\end{aligned}
$$

From (4.20) and (4.21) we infer that

$$
\begin{aligned}
& \left|([U(0, Y)-U(\lambda / 2, Y)]-[U(0,-d)-U(\lambda / 2,-d)])-\frac{4}{\lambda} \int_{0}^{\lambda / 2} \frac{V(X, Y)}{\sin (k X)} \mathrm{d} X\right| \\
& =2\left|\sum_{n=2 j+1: j \geqslant 0} \alpha_{n}\{\cosh [k n(Y+d)]-1\}-\sum_{n=2 j+1: j \geqslant 0} \alpha_{n} \sinh [k n(Y+d)]\right| \\
& =4\left|\sum_{n=2 j+1: j \geqslant 0} \alpha_{n} \sinh \left(\frac{k n(Y+d)}{2}\right)\left\{\cosh \left(\frac{k n(Y+d)}{2}\right)-\sinh \left(\frac{k n(Y+d)}{2}\right)\right\}\right| \\
& =4\left|\sum_{n=2 j+1: j \geqslant 0} \alpha_{n} \sinh \left(\frac{k n(Y+d)}{2}\right) \exp \left(-\frac{k n(Y+d)}{2}\right)\right| \\
& \leqslant 4 \sqrt{\sum_{n=2 j+1: j \geqslant 0} \alpha_{n}^{2} \sinh ^{2}\left(\frac{k n(Y+d)}{2}\right)} \sqrt{\sum_{n=2 j+1: j \geqslant 0} \mathrm{e}^{-k n(Y+d)}} .
\end{aligned}
$$


Since

$$
\sum_{n=2 j+1: j \geqslant 0} \mathrm{e}^{-k n(Y+d)}=\mathrm{e}^{-k(Y+d)} \sum_{j \geqslant 0} \mathrm{e}^{-2 k j(Y+d)}=\frac{\mathrm{e}^{-k(Y+d)}}{1-\mathrm{e}^{-2 k(Y+d)}}=\frac{1}{2 \sinh [k(Y+d)]}
$$

while (4.11) and (4.14) yield

$$
\begin{aligned}
\sum_{n=2 j+1: j \geqslant 0} \alpha_{n}^{2} \sinh ^{2}\left(\frac{k n(Y+d)}{2}\right) & \leqslant \sum_{n \geqslant 1} \alpha_{n}^{2} \sinh ^{2}\left(\frac{k n(Y+d)}{2}\right) \\
& =\frac{4}{\lambda}\left\|V\left(\cdot \frac{Y+d}{2}\right)\right\|_{L^{2}[0, \lambda / 2]}^{2} \\
& \leqslant m_{+}^{2} \sinh ^{2}\left(\frac{k(Y+d)}{2}\right),
\end{aligned}
$$

we deduce that

$$
\begin{aligned}
& \left|([U(0, Y)-U(\lambda / 2, Y)]-[U(0,-d)-U(\lambda / 2,-d)])-\frac{4}{\lambda} \int_{0}^{\lambda / 2} \frac{V(X, Y)}{\sin (k X)} \mathrm{d} X\right| \\
& \quad \leqslant 2 m_{+} \sqrt{\tanh \left(\frac{k(Y+d)}{2}\right)}, \quad-d \leqslant Y \leqslant Y_{0} .
\end{aligned}
$$

The above inequality estimates the decay rate as $Y \downarrow-d$. For this, using (4.14), we get

$$
0<\frac{4}{\lambda} \int_{0}^{\lambda / 2} \frac{V(X, Y)}{\sin (k X)} \mathrm{d} X \leqslant 2 m_{+} \sinh [k(Y+d)], \quad-d \leqslant Y \leqslant Y_{0},
$$

so that for all $Y \in\left[-d, Y_{0}\right]$ we have

$$
\begin{aligned}
& |[U(0, Y)-U(\lambda / 2, Y)]-[U(0,-d)-U(\lambda / 2,-d)]| \\
& \quad \leqslant 2 m_{+}\left\{\sinh [k(Y+d)]+\sqrt{\tanh \left(\frac{k(Y+d)}{2}\right)}\right\} .
\end{aligned}
$$

Concerning the practical applicability, note that in the shallow-water regime $d \ll \lambda$ we have $0<k\left(Y_{0}+d\right) \leqslant k d \ll 1$, so that $m_{+}$in (4.12) has to be large if we are to accommodate waves of moderate and large amplitude. For this reason, in this case the estimate (4.14) might fail to ensure that the vertical fluid velocity $V$ is small throughout the lower half of the layer $\{-d \leqslant Y \leqslant \eta(X)\}$, and therefore the deviation of the flow from a pure current might be considerable. This shortcoming is prevented at depths $d$ exceeding half of the wavelength. Moreover, observations bear out the fact that at depths in excess of half a wavelength the vertical flow variations are slight, while for smaller depths this is not to be expected. Consequently, the considerations in the present paper provide insight into the dynamics of the flow beneath the surface for wave parameters in the regime

$$
d \geqslant \frac{\lambda}{2}
$$

without restriction on the height parameter. While our estimates remain valid in the shallow-water regime $d \ll \lambda$, their practical usefulness is quite limited in this regime. In this context, it is of interest to consider the case of a Stokes expansion for a wave steepness satisfying $k a \ll 1$, a setting in which the Stokes expansion provides a good 
approximation. For a Stokes wave of wavelength $306 \mathrm{~m}$, propagating in deep water with a flat bed located at a depth of $4000 \mathrm{~m}$, the wave speed provided by the Stokes expansion is $22 \mathrm{~m} \mathrm{~s}^{-1}$. The choice $d=240 \mathrm{~m}$ gives a good approximation for the variations of the horizontal flow velocity in the near-surface layer while beneath it the water is practically still. In contrast to this, choosing $d=120 \mathrm{~m}$ we observe that the horizontal velocity profile near the lower boundary of the near-surface layer presents strong variations.

We conclude our discussion by some quantitative considerations. For ocean swell one has $[\eta(0)-\eta(\lambda / 2)] \leqslant 5 \mathrm{~m}$, so that $d \geqslant 120 \mathrm{~m}$ places us within the realm of $\varepsilon \ll 1$, in which case the dispersion relation (3.11) provides us with the wave speed. In the regime (4.30), a good approximation for the wave speed is $c \approx \sqrt{g \lambda / 2 \pi}$, the approximation error with respect to (3.11) being less than $0.5 \%$. For windgenerated swell in the Pacific Ocean one typically has $\lambda \approx 300 \mathrm{~m}$, cf. the discussion in Constantin (2011), so that the choice $d=250 \mathrm{~m}$ leads to the realistic speed $c \approx 70 \mathrm{~km} \mathrm{~h}^{-1}$ since $2 \pi d / \lambda \approx 5.23$ and for $s>1.75$ we have $\tanh (s)>0.97$. In view of (4.10), the variation of the horizontal fluid velocity at the depth $d$ is reduced by a factor of roughly 50 . On the other hand, for swell in the Atlantic Ocean one typically has $\lambda \approx 120 \mathrm{~m}$, with $[\eta(0)-\eta(\lambda / 2)] \leqslant 3 \mathrm{~m}$. The choice $d=120 \mathrm{~m}$ leads us to the realistic speed $c \approx 50 \mathrm{~km} \mathrm{~h}^{-1}$, with a forty-fold reduction of the horizontal fluid velocity variations at depth $d$.

\section{Acknowledgements}

The author is grateful to the referees for useful suggestions. In particular, one of the referees kindly provided the numerical data for the Stokes expansion discussed just after relation (4.30). The support of the ERC Advanced Grant 'Nonlinear studies of water flows with vorticity' is also acknowledged.

\section{REFERENCES}

AmicK, C. J. 1987 Bounds for water waves. Arch. Rat. Mech. Anal. 99, 91-114.

Buffoni, B. \& Toland, J. F. 2003 Analytic Theory of Global Bifurcation. An Introduction. Princeton University Press.

Clamond, D. 1999 Steady finite amplitude waves on a horizontal seabed of arbitrary depth. J. Fluid Mech. 398, 45-60.

ClAmOND, D. 2012 Note on the velocity and related fields of steady irrotational two-dimensional surface gravity waves. Phil. Trans. R. Soc. Lond. A 370, 1572-1586.

Constantin, A. 2006 The trajectories of particles in Stokes waves. Invent. Math. 166, 523-535.

Constantin, A. 2011 Nonlinear Water Waves with Applications to Wave-current Interactions and Tsunamis, CBMS-NSF Regional Conference Series in Applied Mathematics, vol. 81, SIAM.

Constantin, A. 2013 Mean velocities in a Stokes wave. Arch. Rat. Mech. Anal. 207, 907-917.

Constantin, A. \& Johnson, R. S. 2008 On the non-dimensionalisation, scaling and resulting interpretation of the classical governing equations for water waves. J. Nonlinear Math. Phys. 63, 58-73.

Constantin, A. \& Strauss, W. 2010 Pressure beneath a Stokes wave. Commun. Pure Appl. Maths 63, 533-557.

Drennan, W. M., Hui, W. H. \& Tenti, G. 1992 Accurate calculations of Stokes water waves of large amplitude. Z. Angew. Math. Phys. 43, 367-384.

FEnton, J. D. 1985 A fifth-order Stokes theory for steady waves. J. Waterway Port Coastal Ocean Engng 111, 216-234.

Henry, D. 2008 On the deep-water Stokes wave flow. Int. Math. Res. Not. IMRN 2008, Art. ID rnn 071, 7 pp. 
Plotnikov, P. I. \& Toland, J. F. 2002 The Fourier coefficients of Stokes waves. In Nonlinear Problems in Mathematical Physics and Related Topics, vol. I, pp. 303-315. Kluwer/Plenum.

Stokes, G. G. 1847 On the theory of oscillatory waves. Trans. Camb. Phil. Soc. 8, 441-455.

Toland, J. F. 1996 Stokes waves. Topol. Meth. Nonlinear Anal. 7, 1-48.

UMEYAMA, M. 2012 Eulerian-Lagrangian analysis for particle velocities and trajectories in a pure wave motion using particle image velocimetry. Phil. Trans. R. Soc. Lond. A 370, 1687-1702. 